\title{
Potential cellular and regenerative approaches for the treatment of Parkinson's disease
}

\author{
Emma L Lane \\ Olivia J Handley \\ Anne E Rosser \\ Stephen B Dunnett \\ Brain Repair Group, School \\ of Biosciences, Cardiff University, \\ CFIO 3US, UK
}

\begin{abstract}
Parkinson's disease is most commonly treated with a range of pharmacotherapeutics, with the more recent introduction of surgical techniques including deep-brain stimulation. These have limited capabilities to improve symptoms of the disease in more advanced stages, thus new therapeutic strategies including the use of viral vectors and stem cells are in development. Providing a continuous supply of dopamine to the striatum in an attempt to improve the treatment of motor symptoms using enzymes in the dopamine synthesis and machinery is one approach. Alternatively, there are tools which may serve to both protect and encourage outgrowth of surviving neurons using growth factors or to directly replace lost innervation by transplantation of primary tissue or stem cell-derived dopaminergic neurons. We summarize some of the potential therapeutic approaches and also consider the recent EU directives on practical aspects of handling viral vectors, cells and tissues, and in the running of clinical trials in Europe which impact on their development.
\end{abstract}

Keywords: transplantation, viral vector, stem cells, ethics, European Union directive

Parkinson's disease (PD) is one of the most common neurodegenerative diseases affecting around $1 \%-2 \%$ of the population over 70 years of age (MacDonald et al 2000). Motor symptoms are typically first observed as tremor in one extremity; the disease then continues its relentless progression throughout the body. It causes not only the cardinal symptoms of resting tremor, postural instability, rigidity, bradykinesia, and akinesia but also many other symptoms including autonomic dysfunction, depression, and sleep disturbances which can present prior to the motor disorder (Chaudhuri et al 2006). Many of the motor symptoms are ascribed to the specific deterioration of dopaminergic neurons of the nigrostriatal pathway that typifies the disease. Pigmented cell bodies residing in the A9 substantia nigra region of the hindbrain with heavily branched projections targeted mainly at the putamen degenerate retrogradely, striatal innervation being lost first, followed by cell body deterioration. Additional pathology in specific neurochemical systems is observed in the loss of serotonergic, noradrenergic, and cholinergic cells. The pathological criteria for diagnosis are cellular protein inclusions termed Lewy bodies (Jellinger 1991). The extent of these varies enormously from patient to patient and may include areas outside of the nigrostriatal system, for example, the cortex.

The primary therapeutic antiparkinsonian approach in the clinic at present is pharmaceutics-based. Symptomatic treatments are highly effective at managing the motor consequences of nigrostriatal loss, especially in the early stages. However, there is currently no conclusive evidence that any drug licensed for PD has the ability to prevent the relentless wave of degeneration the disease typifies. Ergot and non-ergot derived dopamine $\mathrm{D}_{2}$ receptor agonists and L-dopa, the metabolic precursor to dopamine 
(with a peripheral decarboxylase inhibitor), constitute the mainstay therapies, although there are also a number of adjunctive therapies in use. Over recent years, there has been increasing interest in surgical approaches, which are now in routine use for specific symptoms in many countries. Previously ablative surgeries such as pallidotomy or subthalamotomy have been successfully performed and found to improve certain aspects of motor function. However, this has generally now been replaced by the more easily refined approach of deep-brain stimulation (DBS), involving high frequency stimulation of specific nuclei, most commonly the subthalamic nucleus. While generally effective, these current treatments also have significant inadequacies. The long term use of drug treatments produces multiple sideeffects, not least the 'wearing off' phenomena in which the efficacy of the drug wanes, giving way to the development of motor fluctuation and the onset of abnormal involuntary movements known collectively as 'dyskinesia'. As the disease progresses, ever-increasing doses of L-dopa are required to control the primary symptoms, which exacerbates the side effects, eventually limiting treatment options. There are also symptoms that are non-responsive to drug treatment, such as postural instability and dementia. When applied appropriately DBS can be highly effective at relieving some motor symptoms and reducing the need for L-dopa, but it is costly and generally only considered for patients with late stage disease and without signs of dementia. Furthermore, there is concern about significant side effects such as cognitive decline, which may be permanent.

As a result of these inadequacies, alternative and supplementary symptomatic therapies are still being sought and a number of different pharmacotherapeutic avenues are being explored. These are reviewed in more detail elsewhere (see Schapira et al 2006) but briefly include other potential drugs targeting the dopaminergic system such as dopamine-modulating agents (Ekesbo et al 2000; Pirker et al 2001) and monoamine uptake inhibitors (Frackiewicz et al 2002; Bara-Jimenez et al 2004). Furthermore, drugs affecting the serotonin system, such as the $5-\mathrm{HT}_{1 \mathrm{~A}}$ agonist sarotizan (Bara-Jimenez et al 2005; Goetz et al 2007); adenosine $\mathrm{A}_{2 \mathrm{~A}}$ antagonists; opioids; and glutamatergic and GABAergic drugs are at different stages of development. These compounds can act at several levels: either acting to directly replace the lost dopaminergic transmission at the level of the striatum; acting through alternate mechanisms in the striatum to effect striatal output; or targeting downstream nuclei in the basal ganglia to redress the imbalance in motor output signals.
Some of the aforementioned symptomatic therapies are purported to have neuroprotective potential on the remaining dopaminergic neurones. The rate of progression of the disease on patients taking the selective irreversible monoamine oxidase inhibitors selegiline is slower than those on L-dopa alone suggesting that there is a supplementary action in slowing the degenerative process (Shoulson 1998). However, the data are ambiguous and the design of the clinical trials cannot rule out an entirely symptomatic effect. Multiple mechanisms have been proposed to be involved in the degenerative process, many overlapping with other neurodegenerative diseases. Thus, anti-oxidants, like coenzyme Q10 and vitamins C and E, are being evaluated in different disease conditions for their ability to slow down the disease process.

With the exception of targeted surgical intervention all of the above are systemic therapies, however a generation of potential therapies are under development, which involve more directed cellular approaches to the treatment of PD. In this review, we examine the different methodological approaches to deliver symptomatic, neuroprotective and neurorestorative agents directly to the affected areas of the brain.

The focus of degeneration in PD is the nigrostriatal dopaminergic loss, with the greatest impact being in the loss of input to the striatum. Rebuilding the nigrostriatal tract in an adult in the absence of developmental signals and specific growth signals, has proved highly complex and thus restorative approaches are predominantly focused on placing dopamine cells into the nigrostriatal dopamine neuron target site. Methodological approaches include the use of viral vectors, either directly administered or used to alter cell function prior to transplantation. Furthermore, donor cells can either be transplanted and allowed to integrate with the host environment or encapsulated to facilitate removal. Their development has not only entered novel scientific arenas but has raised practical and ethical concerns which have been addressed at international level with the introduction of new EU legislation to govern these processes. We focus on the areas of this legislation that has greatest practical impact on researchers throughout Europe in the context of these developing therapies.

\section{Symptomatic strategies}

The degeneration of dopaminergic neurons in PD causes the striatum to be depleted of not only dopamine, but also the dopamine synthesizing machinery that is present in terminal axonal regions. There is great interest therefore, in enhancing 
either L-dopa or dopamine production by equipping other cells in the striatum with the necessary 'tools' through the use of viral vectors (Carlsson et al 2007). L-dopa is converted to dopamine either by the remaining dopaminergic terminals or by serotonergic terminals or glia in the striatum (Tanaka et al 1999; Maeda et al 2005). The production of dopamine from L-tyrosine is a multistage process, the rate-limiting enzyme being tyrosine hydroxylase, which converts L-tyrosine to the dopamine precursor L-dopa. However, supplementation with additional tyrosine hydroxylase alone is not a viable approach as its enzymatic functioning requires the presence of a co-factor tetrahydrobiopterin (BH4) (Fukushima et al 1977). Hence, in order to produce functional benefit, a combination of viral vectors expressing both GTP cyclohydrolase 1 (GCH1; a key enzyme in $\mathrm{BH} 4$ synthesis) and tyrosine hydroxylase is required. The advantage of the use of viral vectors in this context is the continuous delivery of dopamine. Continuous enteral infusion of L-dopa or subcutaneous apomorphine significantly reduces motor fluctuations and dyskinesia demonstrating that the pulsatile changes in dopamine levels after oral L-dopa administration may be responsible for their generation (Sage and Mark 1992; Syed et al 1998; Poewe and Wenning 2000; Nyholm et al 2003). Viral vector enhanced production of L-dopa should produce a constant level of dopamine to provide good therapeutic benefit but without the development of dyskinesia. Results in the 6-OHDA lesioned rat demonstrate robust behavioral recovery as assessed by amphetamine-induced rotation and the cylinder test of forelimb asymmetry concurrent with a reduction in L-dopa induced dyskinesia (Carlsson et al 2005). L-dopa delivered to the periphery is subjected to premature conversion to dopamine that necessitates the co-administration of a peripheral amino acid decarboxylase inhibitor (AADC) to increase central uptake of L-dopa. Viral vector mediated enhancement of dopamine synthesis in the striatum would make the use of such supplementary oral drugs redundant. Conversely, it has inspired the use of a triple viral approach delivering AADC intrastriatally in addition to $\mathrm{TH}$ and $\mathrm{GCH} 1$ to facilitate the conversion of L-dopa to dopamine (Sun et al 2004). This approach, using both AAV and lentiviral vectors, improves motor function in rats and primates and increases dopamine levels in the affected striatum (Muramatsu et al 2002).

A potentially simpler approach is to transduce with a viral vector delivering AADC alone (Leff et al 1999). As AADC is lost concomitantly with the degenerating dopaminergic neurons, increasing conversion of L-dopa to dopamine will maximize the efficacy of the absorbed L-dopa. However, this apparently more simple approach could be more risky as there is no inherent feedback mechanism in the control of AADC activity and increased concentrations of AADC could lead to dopamine over production. While TH is inhibited by dopamine, self-limiting dopamine production, AADC has no such innate control mechanism (Carlsson et al 2007). This could lead to even greater degrees of fluctuating striatal dopamine and exacerbate motor disturbances.

One consequence of dopaminergic input in the striatum is disinhibition of the subthalamic nucleus. This is the most common target of DBS, in which high frequency stimulation acts to reduce the activity of this nucleus. Similarly, transfection with an AAV encoding the gene for glutamic acid decarboxylase, synthesizing the inhibitory transmitter GABA has been shown to reduce the excitatory output from the subthalamic nucleus, improving motor cortex activity and consequently in both rodent and macaques models of PD restoring motor function (Luo et al 2002; Lee et al 2005; Emborg et al 2007).

\section{Neurotrophic factors}

In the region of $70 \%-80 \%$ of striatal dopamine is depleted before significant motor symptoms are detected in patients (Bernheimer et al 1973). This suggests that there is a significant pre-symptomatic interval during which some degree of cellular plasticity allows the brain to compensate for the lost innervation. Sprouting of remaining dopaminergic fibres after neurotoxic challenge has been reported in 6-OHDA lesion models of PD, and changes in presynaptic terminals, upregulation of AADC levels to increase dopamine conversions and reduced dopamine transporter have been demonstrated both in MPTP primate models and PD patients. These compensatory mechanisms have the potential to be harnessed to further delay the onset of symptoms or prevent deterioration.

Neurotrophic factors are a family of protein growth factors that regulate components of neuronal growth. Neuron number, branching, axon and dendrite length, neuronal phenotype and synaptic plasticity can all be regulated through their action at specific receptors. Due to the differential expression of these growth factors and their receptors, different neuronal populations are influenced to a greater or lesser extent by each factor. GDNF, neurturin, CNFT and BDNF, to name but a few, have all been evaluated in animal models of PD to determine their effects on dopaminergic neurons. Many molecules have been shown to be neuroprotective, reducing dopaminergic cell death following toxic challenges. GDNF has been the most potent trophic molecule used so far and in animal models of PD has clearly demonstrated a 
capacity to protect neurons against a toxic challenge (Zurn et al 2001; Sherer et al 2006; Patel and Gill 2007). However, these factors require application directly into the relevant area as they do not cross the blood-brain barrier and even when delivered directly into the brain, the precise location of the cannula seems critical. The first studies used intracerebroventricular pumps containing GDNF, which failed to improve symptoms and were associated with serious side effects likely to be as a result of the extrastriatal delivery (Kordower et al 1999; Nutt et al 2003).

The first successful application of GDNF was in the form of a pump installed in the abdomen that released GDNF into the putamen via a thin cannula. The first intraputaminal phase I open label safety trial on 5 patients produced dramatic improvements in motor function in all of the patients (Gill et al 2003). However, a double-blind placebo-controlled trial sponsored by Amgen designed to further examine the benefits of this approach was prematurely terminated (Lang et al 2006). The primary outcome measures had not been achieved and anti-GDNF antibodies had been found in some patients indicating a possible autoimmune response. Furthermore, very high doses of GDNF administered to primates had produced cerebellar lesions. Various technicalities have been given for the failure of this trial including the efficiency of delivery due to use of different cannulae from the initial trial (Lang et al 2006).

Since the potential of GDNF has been determined, there have been alternative approaches to the delivery of the growth factor. The use of viral vectors to engineer cells to produce GDNF within the striatum is being considered to produce a continuous supply, rather than the re-fills required by a pump. The viral vector could be injected directly in vivo transfecting the host striatal cells and enabling them to express GDNF. In 6-OHDA lesioned rats, transfection of adeno-associated viral vectors expressing the GDNF protein have significantly protected dopaminergic terminals in the striatum from insult by 6-OHDA in both rodents and non-human primates (Choi-Lundberg et al 1998; Kirik et al 2000; Wang et al 2002; Eslamboli et al 2003). Problems with this approach include control of the expression of the vector should there be side effects. It has already been demonstrated that aberrant sprouting of fibres and downregulation of tyrosine hydroxylase expression occurs (Georgievska et al 2002; Rosenblad et al 2003) which could potentially limit functional recovery in the long term. Other possible approaches are to transfect cells in vitro and either transplant these GDNF producing cells directly or, more safely, deliver encapsulated cells in a semi-permeable membrane. Such encapulsated GDNF-producing cells have protected nigrostriatal dopaminergic neurons from 6-OHDA toxicity (Shingo et al 2002; Yasuhara et al 2005) and also shown a neurorestorative action by increasing dopamine fibre density, an effect that was sustained following capsule removal (Sajadi et al 2006). The advantages to this approach are that multiple devices could be used to provide GDNF throughout the putamenal area, the lower levels delivered in this approach should minimize side effects and the risks of cerebellar toxicity and antibody generation highlighted by the Amgen trial. As with direct viral vector administration, long-term safety trials firstly in non-human primates need to be carried out to assess the ease of implantation and retrieval, efficacy and also toxicity and immunogenicity (Lindvall and Wahlberg 2008). One disadvantage of this approach concerns the fact that capsules have a limited lifespan and therefore surgery would need to be repeated.

Other growth factors of the same family, neurturin and CDNF, have also been proposed as potential therapeutics. Both growth factors protect dopaminergic neurons from toxic insult in vivo (Rosenblad et al 1999; Li et al 2003; Lindholm et al 2007) but while CDNF is still in the early stages of preclinical studies, neurturin has progressed to clinical trials using intrastriatal delivery through an adeno-associated virus type II (AAV2). Preliminary safety and efficacy data from the phase I clinical trial of 12 patients are encouraging with no significant side effects and an improvement of up to $36 \%$ in the UPDRS score in 9 of the 12 patients (Marks 2006) and phase II trials are underway.

\section{Cell transplantation}

Transplantation of primary ventral mesencephalic tissue into the striatum aims to restore brain circuitry and function lost as a result of PD. The main objective of primary tissue transplantation has been to provide proof-of-principle that grafted dopaminergic neurons can i) survive and restore regulated dopamine release, ii) integrate with the host brain to reinstate frontal cortical connections and activation, and iii) lead to measurable clinical benefits together with improved quality of life.

Preclinical work in animal models of PD has shown that grafted dopaminergic neurons, extracted from the developing ventral mesencephalon (VM) can survive, reinnervate the lesioned striatum, and improve motor function (Bjorklund 1992; Herman and Abrous 1994; Winkler et al 2000). Over the past two decades, a series of open-label clinical trials have provided convincing evidence to show that human embryonic nigral neurons taken at a stage of development when they 
are committed to a dopaminergic phenotype can survive, integrate and function over a long time in the human brain. To date, approximately 350 patients with $\mathrm{PD}$ world-wide have received primary tissue transplantation. Based on a small number of carefully controlled clinical trials, there is good evidence of graft survival, with grafted neurons developing afferent and efferent projections with the host neurons. Longterm survival of dopaminergic grafts is possible up to 10 years after transplantation (Piccini et al 1999), and there have been no reported cases of overt immunorejection even after several years of withdrawal from immunosuppression (Olanow et al 2003). Evidence from PET scanning has revealed significant increases in activation in the areas reinnervated by the grafted cells, and longitudinal clinical assessments indicate significant functional recovery for motor control, in some cases for more than 10 years (Dunnett et al 2001; Lindvall and Hagell 2002; Bjorklund et al 2003). In the most successful cases, patients have either reduced dependency for or completely withdrawn from L-dopa treatment. Post-mortem studies similarly show good survival of transplanted neurons and well integrated grafts (Kordower et al 1995).

Two NIH sponsored double-blind placebo-controlled clinical trials have been unable to replicate the scale of these clinical benefits and reported severe graft-induced dyskinesias during "off" phases (Freed et al 2001; Olanow et al 2003). The precise mechanism responsible for these dyskinesias remains unknown but it does not appear to be related to graft overgrowth resulting in excessive dopamine release (Hagell et al 2002; Lane et al 2006). One possibility surrounds the quality of dissected tissue. Successful trials have used either freshly dissected tissue or tissue that has been stored in culture for only a few days. One of the trials reporting cases of severe dyskinesias used tissue stored in culture for up to four weeks (Freed et al 2001) and it may be that holding tissue in this way reduces its dopaminergic composition (Hagell et al 2002). A further issue concerns the identification of dense hyperdopaminergic areas within the graft of some patients with graft-induced dyskinesias (Ma et al 2002). This may have caused uneven striatal innervation and excessive dopamine release into non-reinnervated areas (Winkler et al 2005). Finally, it should be noted that these studies were severely under-powered, and moreover, subsequent follow-up data have suggested some evidence of efficacy.

It is also possible that variable side effects of graftinduced dyskinesias are related to patient selection. Greater functional improvement is associated with younger patients, and in patients with less advanced disease. This is most likely because the neuropathology is relatively confined to the nigro-striatal pathway and may have better trophic support compared to patients with more advanced disease. Furthermore, patients with more advanced disease and who have become dyskinetic from long-term use of L-dopa may have increased susceptibility to developing graft-induced dyskinesias (Piccini et al 2005; Lane et al 2006).

VM grafts have thus far been placed in the putamen, and patients who received most benefit from these transplants have had pathology restricted to this area both prior to and after transplantation (Piccini et al 2005). Given the range of symptoms and pathology with different levels of severity seen in PD patients it has been proposed that placement of VM grafts should be based on patient-specific profiles determined by FD-PET imaging (Winker et al 2005). The findings observed in the NIH sponsored trials have led to stimulating and extensive review of the different methodological approaches used by different centers to procure, handle and store fetal tissue. It is possible that differences between these approaches may account for the variability in graft-induced functional recovery. A recent review by Winkler et al (2005) identifies the following aspects requiring harmonisation ahead of further clinical trials: dissection of the tissue, the age of donor foetus, the length and type of storage after dissection, the method of dissociation prior to grafting (pieces vs suspension), and the composition of medium used for storage and/or implantation. The limited availability of tissue and the need for multiple donors for single patients leads to problems of co-ordinating and storing tissue prior to transplantation. The quality of the tissue is intrinsically variable and reaching consistent standards of reproducible preparation, quality control and safety assessment to compare accurately the safety and efficacy of this therapy across different trials are very difficult to achieve through this source of tissue. Therefore, while the use of primary tissue has allowed the proof-of-principal studies to be completed, permitting the accumulation of a substantial body of experience, they have also reinforced the fact that alternate source of cells are necessary for transplantation to become a viable therapeutic option.

\section{Stem cells}

Stem cells could provide one such source and would overcome the issue of limited availability of fresh primary fetal cells. A wide range of stem cells are being investigated as potential sources of dopaminergic neurons for transplantation and while it is beyond the scope of this review to go through each of these in detail we will give a short update as to the 
progress being made (for more detailed review see Dass et al 2006; Morizane et al 2008; Zietlow et al 2008). Stem cells can be obtained from various sources including those derived from the early developing embryo (embryonic stem cells), or later in development from gonadal regions (embryonic germ cells), from the developing or adult brain (neural stem cells) and other tissues such as bone marrow, umbilical cord and amniotic fluid and very recently, the carotid body (mesenchymal stem cells). The majority of research thus far with respect to the formation of dopaminergic neurons for the treatment of PD is in embryonic stem cells and neural stem cells. Dopaminergic neurons are more easily obtained from neural stem cells in the developing VM than other parts of the developing central nervous system but the number of dopaminergic cells produced is still very low. Despite genetic manipulation and the addition of various growth and differentiation factors, generating large numbers of dopaminergic cells from this cell type has had mixed results (Wang et al 2004; Yang et al 2004). However, greater success has been achieved with the more complex embryonic stem cells. Derived from blastocysts donated following in vitro fertilization these cells are truly pluripotent. Promising data have been obtained with dopaminergic neurons derived from mouse ES cells, significantly improving motor function in a rat model of PD (Kim et al 2002). However, directing the differentiation of human ES cells has proved complex and while $50 \%$ of cells spontaneously differentiate into neurons upon LIF withdrawal, few are dopaminergic. Thus, there is the need to develop protocols to 'direct' differentiation. The most successful published protocols describe multiple culture stages in which different transcription and growth factors are added at controlled time points (Perrier et al 2004; Roy et al 2006). However, despite good yield of dopaminergic neurons in vitro, clinically relevant long-term survival and behavioral recovery in animal models rivalling that of primary tissue has yet to be convincingly demonstrated (Ben-Hur et al 2004; Park et al 2005; Brederlau et al 2006; Roy et al 2006). The use of embryonic stem cells is still ethically controversial and there is a risk of unregulated growth into undesirable cell types or tumor formation. Neuronal stem cells carry less risk in this respect but furthermore, unlike embryonic stem cells, which are only derived from the embryonic blastocyst, neural stem cells can be found both in embryonic neural tissue and also in specific neurogenic regions of the adult brain. If the in vivo survival of neural stem cells can be improved they hold the potential to provide autologous transplantation as patients provide the cells for their own recovery. Cell lines that also hold that potential and that could be obtained through less invasive means are carotid body and bone marrow derived mesenchymal stem cells.

Interestingly, stem cells may not just be useful as dopamine factories in the striatum. Recent studies in both rodent and primate models have shown significant behavioral recovery following transplantation with neural stem cells. In addition to the generation of a small population of dopaminergic neurons other cells within the graft were found to be releasing growth factors which are purported to exert neuroprotective or neuroregenerative influences (Redmond et al 2007; Yasuhara et al 2007). While more evidence needs to be accumulated on the longevity of this effect, it broadens the potential of neural stem cells from simple dopamine replacement to preserving and enhancing remaining dopaminergic neurons.

\section{Neurogenesis}

As mentioned above endogenous stem cells are present in specific regions of the brain. While the occurrence of neurogenesis in the striatum and substantia nigra is debated (Zhao et al 2003; Frielingsdorf et al 2004), one indisputable neurogenic region is the subventricular zone (SVZ) lying adjacent to the striatum. The cells in the region are an assortment of stem and progenitor cells that have the potential to be mobilized and induced to differentiate by the presence of growth factors or other small molecules. In the normal condition $75 \%-99 \%$ or the cells differentiate into granular GABAergic neurons, with the rest forming periglomular neurons expressing either tyrosine hydroxylase or GABA. The control of proliferation and mobilisation of these cells may be dopaminergic as both MPTP and 6-OHDA mediated dopamine depletion reportedly decrease proliferation in this zone (Baker et al 2004; Hoglinger et al 2004). An additional source of endogenous source of new dopaminergic neurons may be in the recently described presence of tyrosine hydroxylase positive cell bodies in the striatum, which increase in quantity with dopaminergic denervation (Dubach et al 1987; Betarbet et al 1997; Porritt et al 2000). This is believed to be a phenotypic switch in striatal neurons in response to low dopamine levels (Tande et al 2006). As yet there are no imminent therapeutic strategies heading towards the clinic that manipulate these endogenous systems but their potential is waiting to be harnessed. Therapeutic strategies to increase striatal dopamine could involve recruiting newly produced neurons in the SVZ and encouraging them to migrate into the striatum and differentiate into dopaminergic neurones or to stimulate cells resident in the striatum. In order for this to be achieved understanding more about these two processes 
of neurogenesis and phenotypic switching in the striatum is necessary, determining the intrinsic or extrinsic factors responsible may provide an alternative set of mechanisms that could be utilized to treat PD.

\section{Clinical trials of new therapies}

Clinical trials of restorative therapy in PD must follow clinical and research governance frameworks to protect and promote the health and well-being of the patient at all times. Successful frameworks are structured around three main aims: establishing clear lines of responsibility and accountability, implementing the highest possible standards of clinical care, and promoting a constant dynamic of improvement. Within the European Union (EU), legislative acts known as EU directives, require EU member states to achieve a particular result without laying down the means of accomplishing that result, leaving the individual members to devise their own advisory and enforcement bodies. Here we describe two EU directives that need to be considered in clinical trials of restorative therapies for PD, whether of cell transplantation or gene therapy.

\section{EU directive 200 I/20/EC relating to the implementation of good clinical practice in the conduct of clinical trials on medicinal products for human use}

The purpose and scope of this directive is to enhance the protection of human subjects enrolled in clinical trials of medicinal products, to ensure quality of conduct, and to standardize regulation and conduct of clinical trials across Europe. Clinical trials of medicinal products should adhere to current legislation, regulation and guidance including the principles of Good Clinical Practice (GCP); a set of internationally harmonized guidelines to serve the protection of human rights, safety and dignity whilst also assuring ethical and scientific integrity. The Directive details both ethical and scientific quality requirements for all levels of developing a clinical trial for a medicinal product from designing, conducting, recording and reporting in phase I-IV trials. The Directive emphasizes that the objective of a clinical trial is to establish safety and efficacy a therapeutic intervention using one or more medicinal products. A clinical trial must obtain favorable opinion from a legally constituted Ethical Review Board. The conduct of a clinical trial should include statutory inspections by authorities able to verify levels of efficacy and safety that are acceptable and compliant with GCP. Within Europe, the pharmacovigilance system Eudravigilance activates the immediate cessation of a trial with an unacceptable level of risk, exchanging information between Member States using shared databases. The possibility of adverse reactions means that sponsors of clinical trials must be able to provide insurance or indemnity to cover liability costs. Manufacturing and document archiving are further legislated in EU DIRECTIVE 2005/28/EC.

\section{EU DIRECTIVE 2004/23/EC on setting standards of quality and safety for the donation, procurement, testing, processing, preservation, storage, and distribution of human tissues and cells}

The use of human tissues and cells for transplantation has become a strong focus for therapeutic intervention in PD. The Directive only applies to the use of human tissue and cells used for clinical trials to the human body; it does not apply to in vitro research experiments or use in animal models of disease. The quality and safety of these substances must be maintained, in particular to prevent transmission of disease. Donated tissues and cells must be procured, tested, processed, preserved, and stored in accordance with validated and approved safety measures. In order to regulate the highest possible quality control and assurance surrounding the handling, preparation and storage of these substances for use in clinical trials, laboratory conditions must achieve Good Manufacturing Practice (GMP) standards. GMP sets out highly specified conditions of laboratory practice and regulation put in place to ensure successful production and quality control of the therapeutic product. In light of the EU directive, the UK Human Tissue Act (2004) sets out a new legal framework for the storage and use of tissue from the living, and the removal, storage and use of tissues and organs from the dead. The Human Tissue Authority (HTA; www.hta.gov.uk) is the regulatory body for all matters concerning the removal, storage, use and disposal of human tissue for scheduled purposes. The HTA provides advice and guidance, issues codes of practice and is responsible for licensing establishments. The fundamental principle of the HTA is that of consent in relation to the retention and use of living patients' organs and tissue for particular purposes beyond their diagnosis and treatment, and consent surrounding the removal, retention and use of tissue from those who have died (where consent is obtained either by those individuals in life, or after death by someone nominated 
by or close to them). The practicalities of this legislation are that GMP standards have to be adhered to in the handling and processing of human tissue for transplantation. As such, European groups intending to perform transplantations have had to install (at their own cost) facilities and equipment and provide training to gain GMP approval. There is a vastly more detailed paper trail that will ensure the tracking of individual samples from derivation at elective termination to eventual transplantation, and processes previously validated have to be amended to ensure that all products involved in the processing of the tissue are of GMP status.

\section{Considerations in the development of treatments for PD}

Beyond the legislative constraints that arise for all disease treatment strategies using at viral vector or human tissue methodologies, there are practical and ethical issues pertaining to the treatment of PD that require consideration.

\section{Conclusive diagnosis}

A major concern with the treatment of motor disorders and the successful evaluation of future therapies in small scale clinical trials is that there is no conclusive pre-mortem diagnosis of PD. Lewy bodies remain the defining pathological hallmark in addition to the presence of nigrostriatal degeneration, the presence of which cannot be confirmed until post-mortem. Unfortunately the motor symptoms alone are unable to provide a conclusive diagnosis of PD as there is a broad spectrum of phenotypes and a distinct overlap with Parkinson's plus syndromes, even responsiveness to L-dopa, typical of PD, can be confounding in the case of multiple systems atrophy, as early stage patients can present both with symptoms indistinguishable from PD and a responsiveness to L-dopa. Furthermore, even with a confident diagnosis of $\mathrm{PD}$ this in itself incorporates a range of disease phenotypes which may correspond to subgroups of patients for whom different treatment approaches may be more appropriate or more effective (Foltynie et al 2002).

\section{When to intervene?}

The stage of the disease at which the trials should be carried out is an ongoing debate. Where existing therapies are capable of alleviating symptoms it is more complex to involve these patients in clinical trials of highly novel therapeutic approaches. However, patients in a more advanced state may not be the optimum patient group to benefit from the treatment strategy. Inclusion criteria for trials of transplantation of embryonic cells have included a minimum of 7 years of treatment and the presence of intractable problems which indicates patients in the more advanced stages of disease. All these patients had been taking L-dopa for several years and therefore had established L-dopa induced dyskinesia. Rodent experiments have demonstrated an association between the severity of these behaviors or exposure to L-dopa prior to transplantation with the extent of graft-induced dyskinesia (Lane et al 2006). Earlier stage patients are less likely to have developed intractable L-dopa induced dyskinesia and therefore this may be a more suitable target group of patients.

\section{Risks and predicted side effects}

The risks to patients undergoing primary or stem cell therapy must all be considered and eliminated or minimized in preclinical experimentation prior to initiation of a clinical trial, however this is not possible. Some patients that received dopaminergic fetal tissue transplants into the striatum developed the unpredicted side effect of 'off' medication dyskinesia. While the reasons for this side effect are becoming clearer it heightens the need for careful observation to identify unexpected side effects both on and off medication. This development has hindered the progress of primary and stem cell transplantations and will also impact upon viral vector clinical trials. Other risks to patients undergoing cell transplantation include the risk of tumor formation, the migration of cells to inappropriate brain regions, immune rejection of the cells in addition to the inherent risks of neurosurgery such as hemorrhage and post-operative infection.

Gene therapy carries its own associated risks, and although huge progress has been made in recent years, improvements in the safety of viral vectors have to be demonstrated. The risks include insertional mutagenesis associated with retroviral vectors (implicated in the development of leukemia in X-SCID sufferers in France). This is avoided with the use of adeno-associated viruses but the potentially high immunogenicity has also led to recommendations from the UK Gene Therapy Advisory Committee (GTAC) that more detailed immune status and cytokine profiling of the patients is carried out throughout the trial (Relph et al 2004). The first trials carried out with the AAV2 containing neurturin have not demonstrated any adverse side effects, however this only encompasses a small group of patients followed over a short period of time. Unlike cell transplantation, there have been no proof-of-principle experiments helping guide new clinical trials and long-term safety and efficacy trials still need to be carried out. The recent EU directives on good practice in clinical trials and 
the manufacture of products for clinical use detailed above have influenced these processes.

\section{Placebo effects and sham surgery}

As with other diseases, there is evidence that this patient group is subject to placebo effects (de la Fuente-Fernandez and Stoess1 2002). This is commonly seen with non-invasive drug treatments but has also been demonstrated with DBS (de la Fuente-Fernandez 2004). The ethics of blinded sham surgery to demonstrate clinical efficacy is also a contentious area with differing opinions from scientists, patients and public health departments (Boer and Widner 2002; Kim et al 2005; Polgar and Ng 2007; Frank et al 2008). Arguments include the level of risk and associated costs of 'unnecessary' surgical procedures against the validity of non-blinded trials which could lead to false positive results, although the acceptability of this approach to members of the public appears to depend on their disease status, those without PD tending to be more willing to risk sham surgery (Frank et al 2008).

\section{Outcome measures}

Restorative therapy in PD aims to reinstate brain circuitry and function lost as a consequence of progressive neurodegenerative disease. The clinically beneficial effect of a therapy is dependent on two main issues: firstly, whether the therapy will survive and replace lost neurons in the place(s) where required, and secondly, whether the therapy can restore normal function effectively. Assessing efficacy depends on sensitive and reliable outcome measures, ideally well-validated clinical and biological markers collected systematically over a longitudinal period. To date, previous and on-going clinical trials of restorative therapy in PD have relied on extensive assessment protocols, eg, cell therapies follow CAPIT: Core Assessment Protocol for Intracerebral Transplantation, capturing longitudinal data on clinical and imaging markers. In spite of such existing protocols, there is a continued demand for the development of more accurate and reliable outcome measures to assess the scientific and economic feasibility of future restorative therapy.

There are many new avenues of therapy on the horizon for the treatment of PD. However, in order for them to successfully reach the clinic as a mainline treatment clinical trials will have to demonstrate proof of principle, safety and efficacy, as the primary fetal tissue studies have done. There is increasing amounts of legislation surrounding the ethical and practical issues which must be considered in the planning of new clinical trials as well as adequate designs to ensure conclusive data are obtained and the true potential of these novel therapies is realized.

\section{Disclosures}

None of the authors has any conflicts of interest to disclose.

\section{References}

Baker SA, Baker KA, Hagg T. 2004. Dopaminergic nigrostriatal projections regulate neural precursor proliferation in the adult mouse subventricular zone. Eur J Neurosci, 20:575-9.

Bara-Jimenez W, Bibbiani F, Morris MJ, et al. 2005. Effects of serotonin 5-HT1A agonist in advanced Parkinson's disease. Mov Disord, 20:932-6.

Bara-Jimenez W, Dimitrova T, Sherzai A, et al. 2004. Effect of monoamine reuptake inhibitor NS 2330 in advanced Parkinson's disease. Mov Disord, 19:1183-6.

Ben-Hur T, Idelson M, Khaner H, et al. 2004. Transplantation of human embryonic stem cell derived neural progenitors improves behavioral deficit in Parkinsonian rats. Stem Cells, 22:1246-55.

Bernheimer H, Birkmayer W, Hornykiewicz O, et al. 1973. Brain dopamine and the syndromes of Parkinson and Huntington. Clinical, morphological and neurochemical correlations. J Neurol Sci, 20:415-55.

Betarbet R, Turner R, Chockkan V, et al. 1997. Dopaminergic neurons intrinsic to the primate striatum. $J$ Neurosci, 17:6761-8.

Bjorklund A. 1992. Dopaminergic transplants in experimental parkinsonism: cellular mechanisms of graft-induced functional recovery. Curr Opin Neurobiol, 2:683-9.

Bjorklund A, Dunnett SB, Brundin P, et al. 2003. Neural transplantation for the treatment of Parkinson's disease. Lancet Neurol, 2:437-45.

Boer GJ, Widner H. 2002. Clinical neurotransplantation: core assessment protocol rather than sham surgery as control. Brain Res Bull, 58:547-53.

Brederlau A, Correia AS, Anisimov SV, et al. 2006. Transplantation of human embryonic stem cell-derived cells to a rat model of Parkinson's disease: effect of in vitro differentiation on graft survival and teratoma formation. Stem Cells, 24:1433-40.

Carlsson T, Bjorklund T, Kirik D. 2007. Restoration of the striatal dopamine synthesis for Parkinson's disease: viral vector-mediated enzyme replacement strategy. Curr Gene Ther, 7109-20.

Carlsson T, Winkler C, Burger C, et al. 2005. Reversal of dyskinesias in an animal model of Parkinson's disease by continuous L-DOPA delivery using rAAV vectors. Brain, 128:559-69.

Chaudhuri KR, Healy DG, Schapira AH. 2006. Non-motor symptoms of Parkinson's disease: diagnosis and management. Lancet Neurol, 5:235-45.

Choi-Lundberg DL, Lin Q, Schallert T, et al. 1998. Behavioral and cellular protection of rat dopaminergic neurons by an adenoviral vector encoding glial cell line-derived neurotrophic factor. Exp Neurol, 154:261-75.

Clarke CE, 2002. Medical management of Parkinson's disease. J Neurol Neurosurg Psychiatry, 72(Suppl 1):I22-I27.

Dass B, Olanow C W, Kordower JH. 2006. Gene transfer of trophic factors and stem cell grafting as treatments for Parkinson's disease. Neurology, 66:S89-103.

de la Fuente-Fernandez R. 2004. Uncovering the hidden placebo effect in deep-brain stimulation for Parkinson's disease. Parkinsonism Relat Disord, 10:125-7.

de la Fuente-Fernandez R, Stoess1 AJ. 2002. The placebo effect in Parkinson's disease. Trends Neurosci, 25:302-6.

Dubach M, Schmidt R, Kunkel D, et al. 1987. Primate neostriatal neurons containing tyrosine hydroxylase: immunohistochemical evidence. Neurosci Lett, 75:205-10.

Dunnett SB, Bjorklund A, Lindvall O. 2001. Cell therapy in Parkinson's disease - stop or go? Nat Rev Neurosci, 2:365-9. 
Ekesbo A, Andren PE, Gunne LM, et al. 2000. Motor effects of (-)-OSU6162 in primates with unilateral 6-hydroxydopamine lesions. Eur J Pharmacol, 389:193-9.

Eslamboli A, Cummings RM, Ridley, et al. 2003. Recombinant adenoassociated viral vector (rAAV) delivery of GDNF provides protection against 6-OHDA lesion in the common marmoset monkey (Callithrix jacchus). Exp Neurol, 184:536-48.

Emborg ME, Carbon M, Holden JE, et al. 2007. Subthalamic glutamic acid decarboxylase gene therapy: changes in motor function and cortical metabolism. J Cereb Blood Flow Metab, 27:501-9.

Foltynie T, Brayne C, Barker RA. 2002. The heterogeneity of idiopathic Parkinson's disease. J Neurol, 249:138-45.

Frackiewicz EJ, Jhee SS, Shiovitz TM, et al. 2002. Brasofensine treatment for Parkinson's disease in combination with levodopa/carbidopa. Ann Pharmacother, 36:225-30.

Frank SA, Wilson R, Holloway RG, et al. 2008. Ethics of sham surgery: Perspective of patients. Mov Disord, 23:63-8.

Freed CR, Greene PE, Breeze RE, et al. 2001. Transplantation of embryonic dopamine neurons for severe Parkinson's disease. $N$ Engl J Med, 344:710-9.

Frielingsdorf H, Schwarz K, Brundin P, Mohapel P, 2004. No evidence for new dopaminergic neurons in the adult mammalian substantia nigra. Proc Natl Acad Sci U S A, 101:10177-82.

Fukushima K, Richter WE Jr, Shiota T, 1977. Partial purification of 6-(D-erythro-1',2',3'-trihydroxypropyl)-7,8-dihydropterin triphosphate synthetase from chicken liver. $J$ Biol Chem, 252:5750-5.

Georgievska B, Kirik D, Bjorklund A. 2002. Aberrant sprouting and downregulation of tyrosine hydroxylase in lesioned nigrostriatal dopamine neurons induced by long-lasting overexpression of glial cell line derived neurotrophic factor in the striatum by lentiviral gene transfer. Exp Neurol, 177:461-74.

Gill SS, Patel NK, Hotton GR, et al. 2003. Direct brain infusion of glial cell line-derived neurotrophic factor in Parkinson disease. Nat Med, 9:589-95.

Goetz CG, Damier P, Hicking C, et al. 2007. Sarizotan as a treatment for dyskinesias in Parkinson's disease: a double-blind placebo-controlled trial. Mov Disord, 22:179-86.

Hagell P, Piccini P, Bjorklund A, et al. 2002. Dyskinesias following neural transplantation in Parkinson's disease. Nat Neurosci, 5:627-8.

Herman JP, Abrous ND. 1994. Dopaminergic neural grafts after fifteen years: results and perspectives. Prog Neurobiol, 44:1-35.

Hoglinger GU, Rizk P, Muriel MP, et al. 2004. Dopamine depletion impairs precursor cell proliferation in Parkinson disease. Nat Neurosci, 7:726-35.

Jellinger KA, 1991. Pathology of Parkinson's disease. Changes other than the nigrostriatal pathway. Mol Chem Neuropathol, 14:153-97.

Kim JH, Auerbach JM, Rodriguez-Gomez JA, et al. 2002. Dopamine neurons derived from embryonic stem cells function in an animal model of Parkinson's disease. Nature, 418:50-6.

Kim SY, Frank S, Holloway R, et al. 2005. Science and ethics of sham surgery: a survey of Parkinson disease clinical researchers. Arch Neurol, 62:1357-60.

Kirik D, Rosenblad C, Bjorklund A, Mandel RJ. 2000. Long-term rAAV-mediated gene transfer of GDNF in the rat Parkinson's model: intrastriatal but not intranigral transduction promotes functional regeneration in the lesioned nigrostriatal system. $J$ Neurosci, 20:4686-700.

Kordower JH, Freeman TB, Snow BJ, et al. 1995. Neuropathological evidence of graft survival and striatal reinnervation after the transplantation of fetal mesencephalic tissue in a patient with Parkinson's disease. N Engl J Med, 332:1118-24.

Kordower JH, Palfi S, Chen EY, et al. 1999. Clinicopathological findings following intraventricular glial-derived neurotrophic factor treatment in a patient with Parkinson's disease. Ann Neurol, 46:419-24.

Lane EL, Winkler C, Brundin P, Cenci MA. 2006. The impact of graft size on the development of dyskinesia following intrastriatal grafting of embryonic dopamine neurons in the rat. Neurobiol Dis, $22: 334-45$.
Lang AE, Gill S, Patel NK, et al. 2006. Randomized controlled trial of intraputamenal glial cell line-derived neurotrophic factor infusion in Parkinson disease. Ann Neurol, 59:459-66.

Lee B, Lee H, Nam YR, et al. 2005. Enhanced expression of glutamate decarboxylase 65 improves symptoms of rat parkinsonian models. Gene Ther, 12:1215-22.

Leff SE, Spratt SK, Snyder RO, Mandel RJ. 1999. Long-term restoration of striatal L-aromatic amino acid decarboxylase activity using recombinant adeno-associated viral vector gene transfer in a rodent model of Parkinson's disease. Neuroscience, 92:185-96.

$\mathrm{Li} \mathrm{H}, \mathrm{He} \mathrm{Z,} \mathrm{Su} \mathrm{T,} \mathrm{et} \mathrm{al.} \mathrm{2003.} \mathrm{Protective} \mathrm{action} \mathrm{of} \mathrm{recombinant} \mathrm{neurturin} \mathrm{on}$ dopaminergic neurons in substantia nigra in a rhesus monkey model of Parkinson's disease. Neurol Res, 25:263-7.

Lindholm P, Voutilainen MH, Lauren et al. 2007. Novel neurotrophic factor CDNF protects and rescues midbrain dopamine neurons in vivo. Nature, 448:73-7.

Lindvall O, Hagell P. 2002. Role of cell therapy in Parkinson disease. Neurosurg Focus, 13:e2.

Lindvall O, Wahlberg LU. 2008. Encapsulated cell biodelivery of GDNF: A novel clinical strategy for neuroprotection and neuroregeneration in Parkinson's disease? Exp Neurol, 209:82-8.

Luo J, Kaplitt MG, Fitzsimons HL, et al. 2002. Subthalamic GAD gene therapy in a Parkinson's disease rat model. Science, 298:425-9.

Ma Y, Feigin A, Dhawan V, et al. 2002. Dyskinesia after fetal cell transplantation for parkinsonism: a PET study. Ann Neurol, 52:628-34.

MacDonald BK, Cockerell OC, Sander JW, et al. 2000. The incidence and lifetime prevalence of neurological disorders in a prospective community-based study in the UK. Brain, 123:665-76.

Maeda T, Nagata K, Yoshida Y, et al. 2005. Serotonergic hyperinnervation into the dopaminergic denervated striatum compensates for dopamine conversion from exogenously administered 1-DOPA. Brain Res, 1046:230-3.

Marks JJ. 2006. Phase 1 study of putaminal gene transfer with adeno-associated virus serotype 2 [AAV2]-neurturin (CERE-120) for Parkinson's disease: preliminary observations. American Academy of Neurology 58th Annual Meeting, San Diego, CA, 2006.

Morizane A, Li JY, Brundin P. 2008. From bench to bed: the potential of stem cells for the treatment of Parkinson's disease. Cell Tissue Res, 333:323-36.

Muramatsu S, Fujimoto K, Ikeguchi K, et al. 2002. Behavioral recovery in a primate model of Parkinson's disease by triple transduction of striatal cells with adeno-associated viral vectors expressing dopaminesynthesizing enzymes. Hum Gene Ther, 13:345-54.

Nutt JG, Burchiel KJ, Comella CL, et al. 2003. Randomized, double-blind trial of glial cell line-derived neurotrophic factor (GDNF) in PD. Neurology, 60:69-73.

Nyholm D, Askmark H, Gomes-Trolin C, et al. 2003. Optimizing levodopa pharmacokinetics: intestinal infusion versus oral sustained-release tablets. Clin Neuropharmacol, 26:156-63.

Olanow CW, Goetz CG, Kordower JH, et al. 2003. A double-blind controlled trial of bilateral fetal nigral transplantation in Parkinson's disease. Ann Neurol, 54:403-14.

Park CH, Minn YK, Lee JY, et al. 2005. In vitro and in vivo analyses of human embryonic stem cell-derived dopamine neurons. J Neurochem, 92:1265-76.

Patel NK, Gill SS. 2007. GDNF delivery for Parkinson's disease. Acta Neurochir Suppl, 97:135-54.

Perrier AL, Tabar V, Barberi T, et al. 2004. Derivation of midbrain dopamine neurons from human embryonic stem cells. Proc Natl Acad Sci U S A, 101:12543-8.

Piccini P, Brooks DJ, Bjorklund A, et al. 1999. Dopamine release from nigral transplants visualized in vivo in a Parkinson's patient. Nat Neurosci, 2:1137-40.

Piccini P, Pavese N, Hagell P, et al. 2005. Factors affecting the clinical outcome after neural transplantation in Parkinson's disease. Brain, 128:2977-86. 
Pirker W, Tedroff J, Ponten H, et al. 2001. Coadministration of (-)-OSU6162 with 1-DOPA normalizes preproenkephalin mRNA expression in the sensorimotor striatum of primates with unilateral 6-OHDA lesions. Exp Neurol, 169:122-34.

Poewe W, Wenning GK. 2000. Apomorphine: an underutilized therapy for Parkinson's disease. Mov Disord, 15:789-94.

Polgar S, Ng J. 2007. A critical analysis of evidence for using sham surgery in Parkinson's disease: implications for public health. Aust NZJ Public Health, 31:270-4.

Porritt MJ, Batchelor PE, Hughes AJ, et al. 2000. New dopaminergic neurons in Parkinson's disease striatum. Lancet, 356:44-45.

Redmond DE Jr, Bjugstad KB, Teng YD, et al. 2007. Behavioral improvement in a primate Parkinson's model is associated with multiple homeostatic effects of human neural stem cells. Proc Natl Acad Sci U S A, 104:12175-80.

Relph K, Harrington K, Pandha H. 2004. Recent developments and current status of gene therapy using viral vectors in the United Kingdom. $B M J$, 329:839-42.

Rosenblad C, Georgievska B, Kirik D. 2003. Long-term striatal overexpression of GDNF selectively downregulates tyrosine hydroxylase in the intact nigrostriatal dopamine system. Eur J Neurosci, 17:260-70.

Rosenblad C, Kirik D, Devaux B, et al. 1999. Protection and regeneration of nigral dopaminergic neurons by neurturin or GDNF in a partial lesion model of Parkinson's disease after administration into the striatum or the lateral ventricle. Eur J Neurosci, 11:1554-66.

Roy NS, Cleren C, Singh SK, et al. 2006. Functional engraftment of human ES cell-derived dopaminergic neurons enriched by coculture with telomerase-immortalized midbrain astrocytes. Nat Med, 12:1259-68.

Sage JI, Mark MH. 1992. The rationale for continuous dopaminergic stimulation in patients with Parkinson's disease. Neurology, 42:23-8; discussion 57-60.

Sajadi A, Bensadoun JC, Schneider BL, et al. 2006. Transient striatal delivery of GDNF via encapsulated cells leads to sustained behavioral improvement in a bilateral model of Parkinson disease. Neurobiol Dis, 22:119-29.

Schapira AH, Bezard E, Brotchie J, et al. 2006. Novel pharmacological targets for the treatment of Parkinson's disease. Nat Rev Drug Discov, 5:845-54.

Sherer TB, Fiske BK, Svendsen CN, et al. 2006. Crossroads in GDNF therapy for Parkinson's disease. Mov Disord, 21:136-41.

Shingo T, Date I, Yoshida H, Ohmoto T. 2002. Neuroprotective and restorative effects of intrastriatal grafting of encapsulated GDNF-producing cells in a rat model of Parkinson's disease. J Neurosci Res, 69:946-54.

Shoulson I. 1998. DATATOP: a decade of neuroprotective inquiry Parkinson Study Group. Deprenyl and tocopherol antioxidative therapy of parkinsonism. Ann Neurol, 44:S160-6.
Sun M, Kong L, Wang X. et al. 2004. Coexpression of tyrosine hydroxylase, GTP cyclohydrolase I, aromatic amino acid decarboxylase, and vesicular monoamine transporter 2 from a helper virus free herpes simplex virus type 1 vector supports high-level, long-term biochemical and behavioral correction of a rat model of Parkinson's disease. Hum Gene Ther, 15:1177-96.

Syed N, Murphy J, Zimmerman T Jr, et al. 1998. Ten years' experience with enteral levodopa infusions for motor fluctuations in Parkinson's disease. Mov Disord, 13:336-8.

Tanaka H, Kannari K, Maeda T, et al. 1999. Role of serotonergic neurons in L-DOPA-derived extracellular dopamine in the striatum of 6-OHDA-lesioned rats. Neuroreport, 10:631-4.

Tande D, Hoglinger G, Debeir T, et al. 2006. New striatal dopamine neurons in MPTP-treated macaques result from a phenotypic shift and not neurogenesis. Brain, 129:1194-200

Wang L, Muramatsu S, Lu Y, et al. 2002. Delayed delivery of AAV-GDNF prevents nigral neurodegeneration and promotes functional recovery in a rat model of Parkinson's disease. Gene Ther, 9:381-9.

Wang X, Lu Y, Zhang H, et al. 2004. Distinct efficacy of pre-differentiated versus intact fetal mesencephalon-derived human neural progenitor cells in alleviating rat model of Parkinson's disease. Int J Dev Neurosci, 22:175-83.

Winkler C, Kirik D, Bjorklund A. 2005. Cell transplantation in Parkinson's disease: how can we make it work? Trends Neurosci, 28:86-92.

Winkler C, Kirik D, Bjorklund A, Dunnett SB. 2000. Transplantation in the rat model of Parkinson's disease: ectopic versus homotopic graft placement. Prog Brain Res, 127:233-65.

Yang M, Donaldson AE, Marshall CE, et al. 2004. Studies on the differentiation of dopaminergic traits in human neural progenitor cells in vitro and in vivo. Cell Transplant, 13:535-47.

Yasuhara T, Shingo T, Date I. 2007. Glial cell line-derived neurotrophic factor (GDNF) therapy for Parkinson's disease. Acta Med Okayama, 61:51-6.

Yasuhara T, Shingo T, Muraoka K, et al. 2005. Early transplantation of an encapsulated glial cell line-derived neurotrophic factor producing cell demonstrating strong neuroprotective effects in a rat model of Parkinson disease. J Neurosurg, 102:80-9.

Zhao M, Momma S, Delfani K, et al. 2003. Evidence for neurogenesis in the adult mammalian substantia nigra. Proc Natl Acad Sci U S A, 100:7925-30

Zietlow R, Lane EL, Dunnett SB, et al. 2008. Human stem cells for CNS repair. Cell Tissue Res, 331:301-22.

Zurn AD, Widmer HR, Aebischer P. 2001. Sustained delivery of GDNF: towards a treatment for Parkinson's disease. Brain Res Brain Res Rev, $36: 222-9$. 
